\title{
Contractile Inactivation in Frog Skeletal Muscle Fibers
}

\author{
The Effects of Low Calcium, Tetracaine, \\ Dantrolene, D-600, and Nifedipine
}

\author{
CARLO CAPUTO and PURA BOLAÑOS
}

From the Laboratorio de Biofísica del Músculo, Centro de Biofisica y Bioquimica, Instituto Venezolano de Investigaciones Cientificas (IVIC), Caracas 1020A, Venezuela

\begin{abstract}
Short muscle fibers $(\sim 1.5 \mathrm{~mm})$ of Rana pipiens were voltageclamped with a two-microelectrode technique at a holding potential of -100 $\mathrm{mV}$. Using conditioning depolarizing ramps, with slopes $>0.2 \mathrm{mV} / \mathrm{s}$, partially inactivated responses are obtained at threshold values between -55 and -35 $\mathrm{mV}$. With slopes equal to or slower than $0.1 \mathrm{mV} / \mathrm{s}$, one inactivates contraction without ever activating it. When the membrane potential is brought slowly to values more positive than about $-40 \mathrm{mV}$, test pulses, applied on top of the ramps, bringing the membrane potential to values up to $+100 \mathrm{mV}$, are ineffective in eliciting contractile responses, which indicates complete inactivation. After inactivation, contractile threshold is shifted by perhaps $10 \mathrm{mV}$, to about $-40 \mathrm{mV}$. The sensitivity of fibers to depolarizing ramps is increased by D-600 $(50 \mu \mathrm{M})$, dantrolene $(50 \mu \mathrm{M})$, tetracaine $(100 \mu \mathrm{M})$, and low calcium $\left(10^{-8} \mathrm{M}\right)$. In the presence of these agents, complete inactivation was obtained using ramp slopes of $1,0.8,0.4$, and $0.2 \mathrm{mV} / \mathrm{s}$, respectively. Nifedipine was less effective. With D-600, once inactivation had been induced, no repriming occurred after repolarization to $-100 \mathrm{mV}$, and partial recovery occurred after washing out the drug. With low calcium, tetracaine, and nifedipine, the tension-voltage relationship was not affected, whereas the steady state inactivation curve (obtained in repriming experiments) was shifted by $10-25 \mathrm{mV}$ toward more negative potentials. With D-600, the activation curve was not modified, whereas the inactivation curve could not be obtained, because of repriming failure. With dantrolene, the inactivation curve was not affected, whereas the activation curve was shifted toward less negative potentials and peak tension diminished, depending on the pulse duration. The results indicate that it is possible to induce complete inactivation without activation, and to differentiate activation and inactivation parameters pharmacologically, which suggests that the two are separate processes.
\end{abstract}

Address reprint requests to Dr. Carlo Caputo, Centro de Biofisica y Bioquimica, Instituto Venezolano de Investigaciones Científicas (IVIC), Laboratorio de Biofisica del Musculo, Apartado 21827, Caracas $1020 \mathrm{~A}$, Venezuela.

J. Gen. Physiol. @ The Rockefeller University Press · 0022-1295/87/03/0421/22 \$1.00

Volume 89 March $1987 \quad 421-442$ 


\section{N T R O D U C TION}

It is well known that, after relaxation from a potassium contracture, skeletal muscle fibers enter a state of contractile refractoriness, which can be reversed by membrane repolarizations through a process termed repriming (Hodgkin and Horowicz, 1960). Contractile inactivation can also be achieved by suprathreshold or subthreshold membrane conditioning depolarizations (Frankenhaeuser and Lannergren, 1976; Caputo and Fernández de Bolaños, 1979; Caputo et al., $1984 b$ ). Since contractile inactivation and repriming are related to the mechanism that regulates calcium release from the sarcoplasmic reticulum (SR) during contractile activation, their study could be important for understanding the latter process. At this point, it is important to note that the term inactivation, as used here, refers to a slow process that takes seconds to develop and is reversed with a slow time course during repriming. This phenomenon is probably different from the faster inactivation described by Baylor et al. (1983) and Melzer et al. (1984). These authors have studied the time course of calcium release, using metallochromic calcium indicators, and have used the term inactivation to describe the fall of the calcium signal that occurs during relatively short pulses $(100-500 \mathrm{~ms})$. In this work, we have tried to induce contractile refractoriness (here called inactivation) without prior activation, and to test whether these two phenomena, activation and inactivation, are the expression of one process or two different processes. When studied with subthreshold conditioning depolarizing pulses in voltage-clamped frog muscle fibers, the inactivation phenomenon is characterized by an initial shortening of the contracture response time course, followed by a reduction of the response peak tension (Caputo et al., 1984b). With this approach, however, it is difficult to dissociate inactivation from activation, since the fibers usually contract, albeit with a diminished response, when the conditioning pulses reach the region of contractile threshold.

In this work, we have employed conditioning depolarizing ramps to induce inactivation. It is shown that with sufficiently slow ramps, it is possible to achieve complete inactivation without activation. It is important to clarify that, since in these experiments isometric tension is the measured contractile parameter, the possibility of a subthreshold calcium release cannot be ignored. With regard to the second point, there are several drugs, or experimental treatments, that affect the process of depolarization-contraction coupling differently (Caputo, 1983). For instance, tetracaine $(1-2 \mathrm{mM})$ and dantrolene are known to interfere with calcium release from the SR, possibly affecting an early step in the coupling process (Huang, 1982; Hui, 1983; Vergara and Caputo, 1983). On the other hand, tetracaine at lower concentrations $(0.1 \mathrm{mM})$ or treatment with low-calcium solutions causes a shortening of the potassium contracture time course, which resembles the effect of incomplete contractile inactivation (Caputo, 1972), and thus suggests an effect on this latter process. More recently, it has been shown that D-600, a known calcium antagonist, may cause paralysis of muscle fibers after the induction of a full-sized potassium contracture (Eisenberg et al., 1983), which also indicates that this drug might interfere with the repriming process. We have tested the effectiveness of low-calcium solutions, tetracaine at a low concentration, dantrolene, and D-600 in altering contractile activation, inacti- 
vation, and repriming. For comparative purposes, the effects of a different calcium antagonist, nifedipine (Cota and Stefani, 1981; Almers et al., 1981), were also studied.

\section{ETHODS}

The experiments described in this work were carried out with bundles of 5-20 fibers dissected from the $\mathrm{m}$. lumbricalis IV digiti of the hind limb of Rana pipiens. These fibers have a short length $(1.5 \mathrm{~mm})$ and can be voltage-clamped with a two-microelectrode technique according to the procedure and with the experimental system already described (Caputo et al., 1984a).

In the present experiments, tension was recorded with a force transducer (series 400 , Cambridge Technology, Cambridge, MA) and displayed on an oscillograph (model 220, Gould, Inc., Cleveland, $\mathrm{OH}$ ).

The conditioning depolarizing ramps were generated either with a voltage calibrator with variable output, from 0 to $200 \mathrm{mV}$, or with a Digitimer D3030 (Hertfordshire, England), whose output, after being divided, was fed into the input of an operational amplifier wired as a relay-resettled integrator with variable characteristic time $T=R \cdot C$, in which $C$ was a $4.7-\mathrm{F}$ polycarbonate condenser, and $R$ was an array of 11 resistors ranging from $27 \mathrm{k} \Omega$ to $18 \mathrm{M} \Omega$. The output of the integrator went to the summing junction of the clamp control amplifier. With this system, ramps with slopes as low as $0.08 \mathrm{mV} / \mathrm{s}$ could be obtained.

\section{Solutions}

The basic solution used in these experiments was a normal Ringer's (NR) of the following composition (in $\mathrm{mM}$ ): $115 \mathrm{NaCl}, 2.5 \mathrm{KCl}, 1.8 \mathrm{CaCl}_{2}, 10 \mathrm{mM}$ Tris buffer, $\mathrm{pH}$ 7.4. Tetrodotoxin was added at a concentration of $10^{-6} \mathrm{~g} / \mathrm{liter}$. The low-calcium solution was prepared without $\mathrm{CaCl}_{2}$, and with $5 \mathrm{mM} \mathrm{MgCl}_{2}$ and $1 \mathrm{mM}$ EGTA-Tris. Tetracaine (Sigma Chemical Co., St. Louis, MO) was added at the desired concentration to the normal medium. Dantrolene sodium, a kind gift of Norwich Laboratories (Norwich, NJ), was either dissolved into dimethyl sulfoxide (DMSO) (E. Merck, Darmstadt, Federal Republic of Germany) and later diluted 2:1,000 in normal Ringer's to a final concentration of 20-50 $\mu \mathrm{M}$, or in other experiments after stirring in the normal solutions overnight in the dark, at a concentration of $30 \mu \mathrm{M}$. When DMSO was used, control experiments showed that it had no effect on the fibers' contractile properties. D-600, kindly provided by Dr. A. Oberdorf, Knoll AG (Ludwigshafen, Federal Republic of Germany), was dissolved by continuous stirring for at least $3 \mathrm{~h}$, in the dark, and used immediately after. Nifedipine (the first batch of which was a kind gift of Enrico Stefani, and was later purchased from Sigma Chemical Co.) was dissolved in ethanol at $50 \mathrm{mM}$ and then diluted to a final concentration of 50-200 $\mu \mathrm{M}$. The stock solutions were stored for few days at $-20^{\circ} \mathrm{C}$. The nifedipine solutions were always kept and used in darkness. The final maximal ethanol concentration was $4.6 \mathrm{mM}$, and was found to have only minor effects on the fibers' contractile properties.

\section{RES U L T S}

\section{Inactivation Process}

Fig. 1 demonstrates the effectiveness of conditioning polarizing ramps for achieving contractile inactivation without previous activation. In $A$, the upper record shows a contracture response (lower trace) to a prolonged depolarization to 0 $\mathrm{mV}$ (upper trace). The two lower records, obtained sequentially without inter- 
$\mathbf{A}$

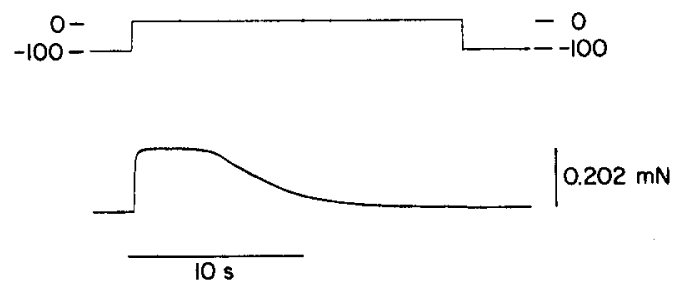

$10 \mathrm{~s}$

$\begin{array}{rr}+100- & -+100 \\ 0- & -20 \\ -100-1\end{array}$

$-100$

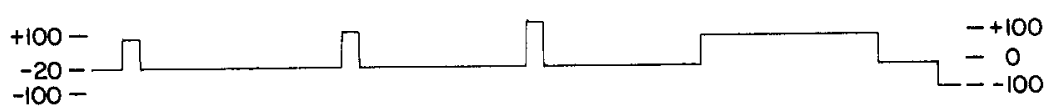

$-100-$

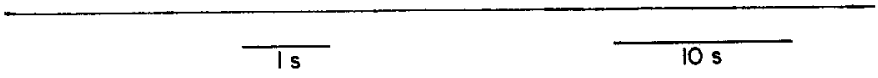

B

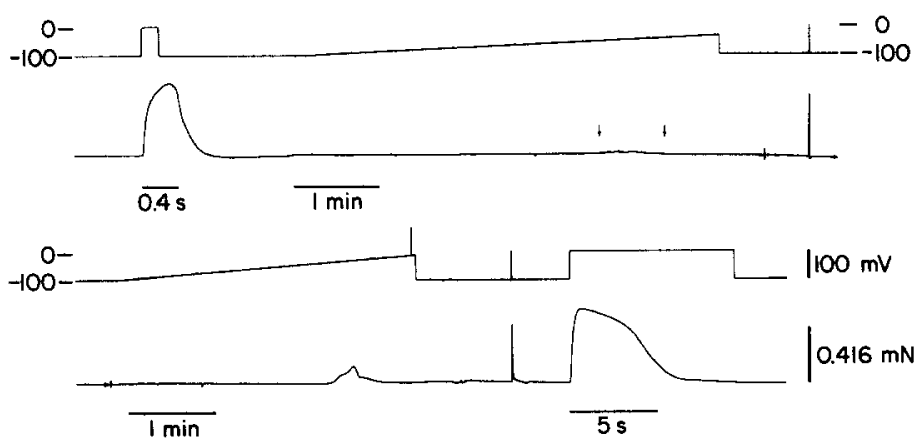

FIGURE 1. Effect of conditioning depolarizing ramps on the tension output of voltage-clamped single muscle fibers. In $A$, the upper record shows the contracture response (lower trace) to a voltage-clamp depolarizing pulse (upper trace) from -100 to $0 \mathrm{mV}$. The second and third records demonstrate the effectiveness of a ramp of $0.11 \mathrm{mV} / \mathrm{s}$ slope in producing complete contractile inactivation. The two records were obtained consecutively. It can be seen that after the membrane had been depolarized to about $-20 \mathrm{mV}$, a positive pulse to about $+80 \mathrm{mV}$ had no effect; further pulses of increasing magnitude and duration were equally ineffective, which indicates complete inactivation. Notice the change in the time base when pulses were applied. Panel $B$ demonstrates that while a ramp of $0.215 \mathrm{mV} / \mathrm{s}$ was barely effective in producing complete inactivation (the arrows indicate a small perturbation in the tension trace), a faster ramp, with a slope of $0.4 \mathrm{mV} / \mathrm{s}$, produced only partial inactivation. The pulses at the beginning and at the end of the ramps showed normal contractile responses. 
ruption and with a slower time base, show that as result of a slow depolarizing ramp of constant slope, $0.11 \mathrm{mV} / \mathrm{s}$, which brought the membrane potential from -100 to $0 \mathrm{mV}$, no tension was developed while the potential spanned this range, or when the fiber was further tested with pulses of different durations, which brought the membrane to positive values up to $+100 \mathrm{mV}$. Panel $B$, obtained with another fiber, demonstrates that the ramp slope value is crucial for achieving complete inactivation. In the upper record, the fiber was first tested with a 200$\mathrm{ms}, 100-\mathrm{mV}$ pulse (notice the faster time base), and was later depolarized with a $0.215-\mathrm{mV} / \mathrm{s}$ slope ramp, which produced only a minor perturbation in the tension trace. In the lower record, a faster ramp, with a slope of $0.4 \mathrm{mV} / \mathrm{s}$, was employed, and a sizable, although reduced, response was obtained when the membrane

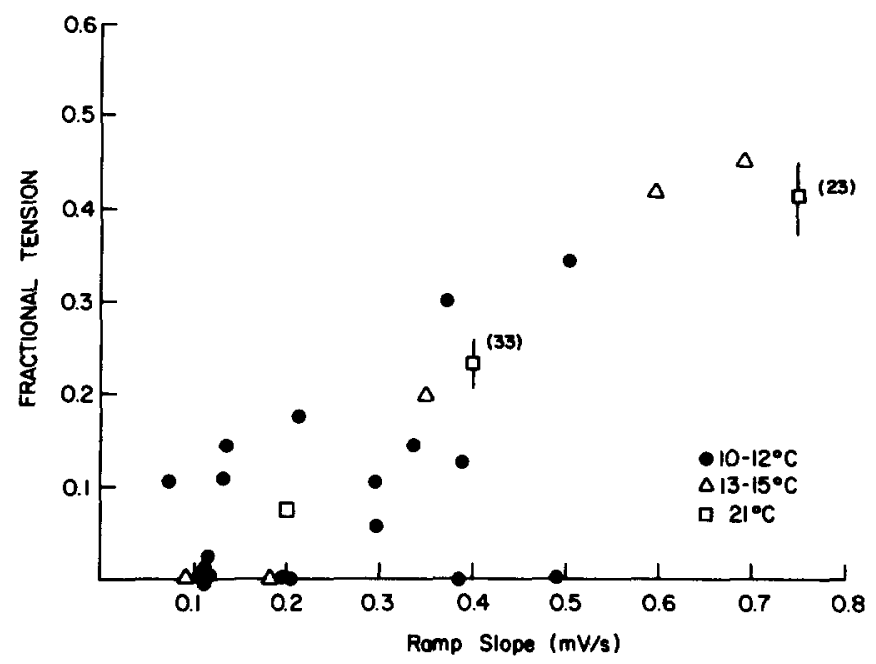

Figure 2. Relation between the extent of inactivation, expressed as fractional tension, and the value of the slope of depolarizing ramps. The fractional tension is referred to control responses induced before applying the depolarizing ramps. The filled circles represent results obtained with individual fibers. The open squares represent the mean of different experiments (numbers are given in parentheses); the SEM is represented by the vertical bars. The experiments were carried out at the temperatures indicated.

potential had reached a value of about $-50 \mathrm{mV}$, which indicated partial inactivation of the fiber.

Fig. 2 summarizes the relationship existing between the degree of inactivation produced by depolarizing ramps and the ramp slope value. In the graph, the tension developed during ramps of varying effectiveness is expressed as a fractional value of the maximal tension of each fiber. The squares represent average values obtained with several fibers (the number is given in parentheses), and the other points correspond to values obtained with individual fibers. The results were obtained at the temperatures indicated. In spite of considerable scatter caused by fiber variability, it is clear that complete inactivation can be obtained using ramps with slopes slower than $0.1 \mathrm{mV} / \mathrm{s}$; above this value, the degree of 
inactivation decreases insofar as the ramp slope values increase. Unfortunately, it was not possible to obtain consistent results with ramps slower than $0.1 \mathrm{mV} / \mathrm{s}$ because of stability problems with both the preparation and the equipment. The loss of contractile responses described above could be due to an effect on the activation mechanism and not necessarily to inactivation. For instance, one could think of a continuous shift of the contractile threshold value toward more positive potentials when the fibers are conditioned with subthreshold depolarizations. However, the unresponsiveness of the fibers, even when tested in the more positive potential regions, argues against this possibility.

Fig. 3 shows the relationship between the contractile threshold and the slope of the ramps employed with several fibers that were only partially inactivated. Thus, the contractile threshold could be determined as the potential where the first signs of tension development could be detected. With this approach, it is probable that an overestimate of the contractile threshold increase would be

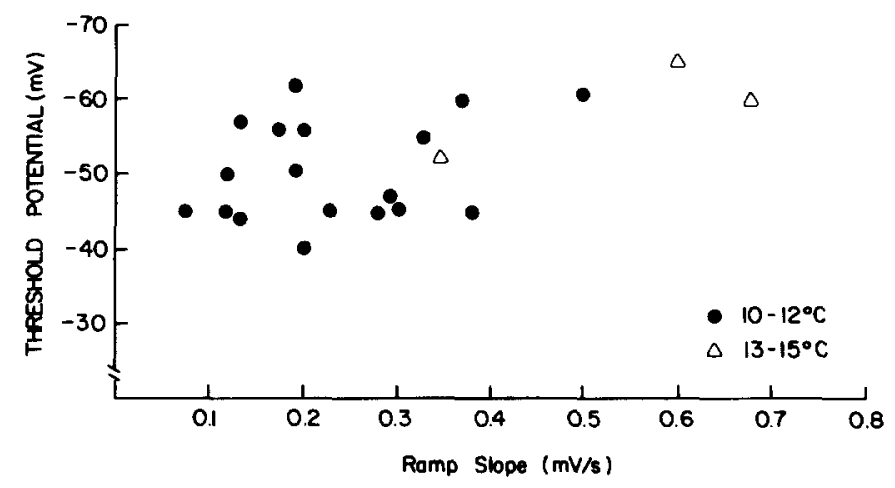

FigurE 3. Relation between contractile threshold and ramp slope values. The contractile threshold was determined as the potential at which the first signs of tension development were observed, in runs in which the depolarizing ramp was not completely effective in producing complete inactivation. The experiments were carried out at the temperatures indicated.

obtained. This is due to the possibility of a delay between the moment at which the contractile threshold is reached during a ramp and the moment at which tension development is detected. Thus, an apparently higher threshold could be obtained with faster ramps. A regression line $(Y=-23.8 \cdot X-45.3 ; r=0.5)$ fitted to the experimental points appears to intercept the potential axis at about $-45 \mathrm{mV}$. This indicates that complete inactivation can be achieved once this value is reached and surpassed, in agreement with the steady state inactivation curves obtained in repriming experiments (Hodgkin and Horowicz, 1960; Lüttgau, 1973).

At this point, it is convenient to explore whether the curves obtained with repriming experiments are similar to those in which inactivation is induced without previous activation. The graph of Fig. 4 shows the relationship between the degree of inactivation and the fiber membrane potential. The degree of inactivation was measured by performing experiments of the type shown in the 
inset of Fig. 4. In these experiments, the contractile responses to a test pulse to $0 \mathrm{mV}$, obtained after a subthreshold conditioning pulse, are normalized with respect to the responses obtained without the conditioning pulse. In the graph of Fig. 4, the degree of inactivation is expressed either as the fractional peak tension or the fractional area under the contracture response. Some of the points of this graph were obtained from a previous work (Caputo et al., 1984b); the point represented by the half-filled symbol represents the value of $-45 \mathrm{mV}$ for complete inactivation obtained from Fig. 3. It is important to stress that this inactivation curve, obtained in experiments in which no contractile activation had occurred, is very similar to the steady state inactivation curves obtained in

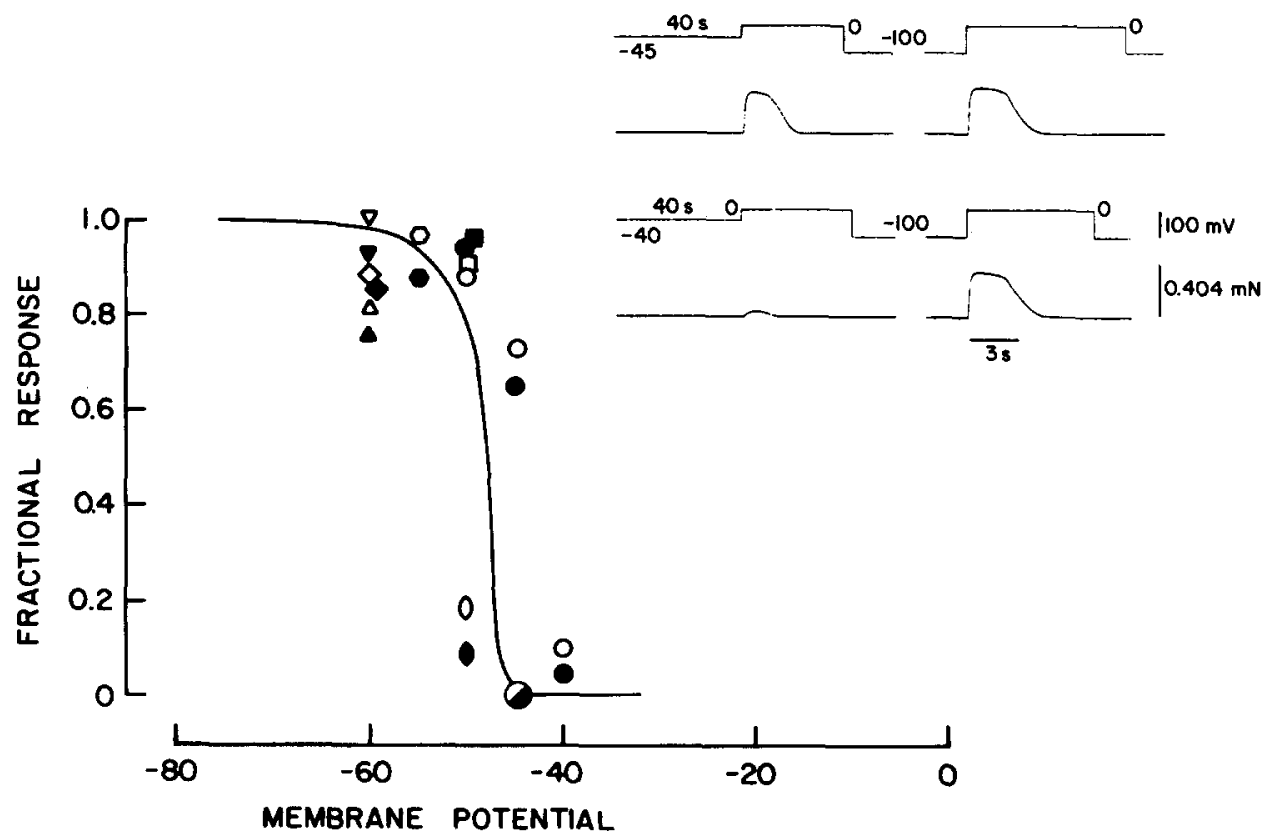

FIGURE 4. Inactivation curve for peak tension (open symbols) and for the area under the contracture response (filled symbols) obtained in experiments in which no tension was developed during the conditioning depolarization. The inset in the upper right shows the protocol of one of these experiments.

repriming experiments under voltage-clamp conditions (Bolaños et al., 1986; Figs. 7, 9, and 13 of this work). One point that has not yet been clarified is whether contractile activation and inactivation can be considered two different responses. In fact, the reduction or absence of contractile responses after conditioning depolarizations (rectangular pulses or ramps) could be interpreted as a failure of the activation process. This point is dealt with next.

\section{Effect of Low Calcium and Tetracaine}

It has been shown that low-calcium solutions or low concentrations (0.1-0.2 $\mathrm{mM})$ of tetracaine shorten the time course of potassium contractures by reducing or 
abolishing the plateau phase and increasing the rate of spontaneous relaxation of these responses (Caputo, 1972). It has been suggested that these effects might be mediated by a facilitation of the inactivation process. Fig. 5 shows that exposure to either low-calcium medium (prepared with $1 \mathrm{mM}$ EGTA and $5 \mathrm{mM}$ $\mathrm{Mg}$ ) or $0.1 \mathrm{mM}$ tetracaine increases the effectiveness of depolarizing ramps in producing complete inactivation in two different fibers. In the upper set of

A NR
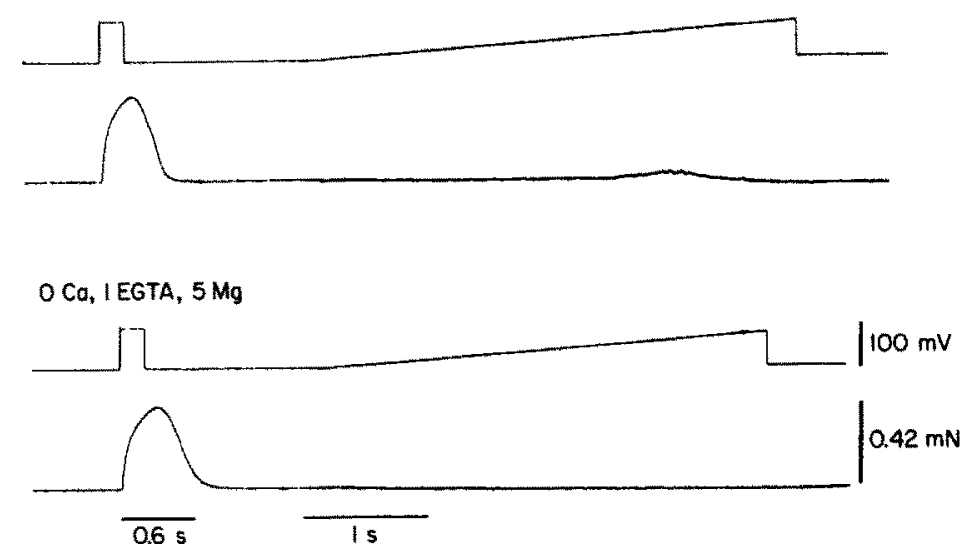

B $100 \mu \mathrm{M}$ Tetracaine NR

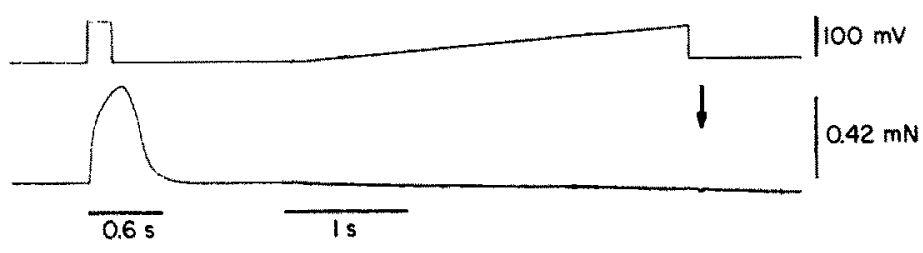

NR

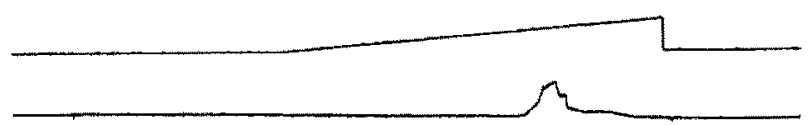

Figure 5. Effect of low external calcium $(A)$ and $100 \mu \mathrm{M}$ tetracaine $(B)$ on the sensitivity of two different fibers to conditioning depolarizing ramps. In both cases, the effectiveness of the conditioning ramp was increased.

records $(A)$, one fiber was first tested in normal solutions with a ramp of 0.4 $\mathrm{mV} / \mathrm{s}$ and gave a small response at about $-45 \mathrm{mV}$. After exposure to a lowcalcium medium for $3 \mathrm{~min}$, a ramp with the same slope was effective in abolishing the contractile response. Panel $B$ shows that, in the presence of $0.1 \mathrm{mM}$ tetracaine, a ramp of $0.75 \mathrm{mV} / \mathrm{s}$ caused complete inactivation, whereas in its absence a sizable response was obtained at about $-50 \mathrm{mV}$. The results of several experi- 
ments of this type are summarized in Fig. $6 \mathrm{~A}$. It is important to note that in the presence of either low calcium or tetracaine, before the ramp, a test stimulus induced a normal response, as shown in the records of Fig. 5; this indicates that the capacity of the fiber to respond to an initial short stimulus is not affected. With respect to this point, it is well established that the absence of external
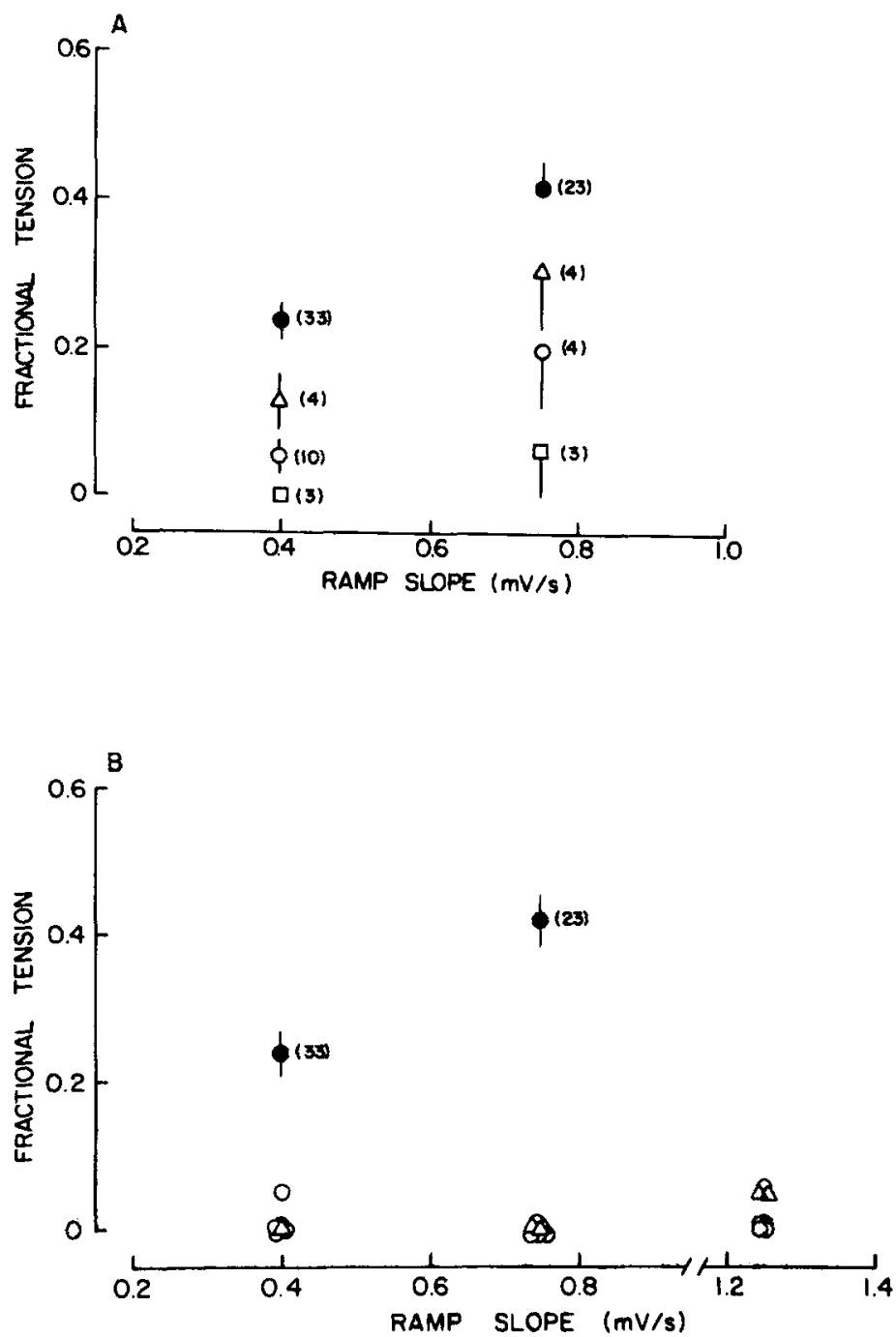

FIGURE 6. Effect of different agents on the fiber sensitivity to depolarizing ramps. The graphs are similar to that shown in Fig. 2, and demonstrate that the effectiveness in facilitating inactivation follows the order: D-600 $(50 \mu \mathrm{M})$, dantrolene $(50 \mu \mathrm{M})$, tetracaine $(100 \mu \mathrm{M})$, low calcium, nifedipine $(50 \mu \mathrm{M})$. $(A) \Theta, N R$ (normal Ringer's); $0,0 \mathrm{Ca}, 5 \mathrm{mM} \mathrm{Mg}, 1 \mathrm{EGTA} ; \Delta$, nifedipine; $\square, 100 \mu \mathrm{M}$ tetracaine. $(B) \bullet, N R ; \Delta$, dantrolene; O, D-600. 
calcium does not impair muscle fibers' capacity to contract, and it causes only a small shift in the tension-voltage relationship but a larger one in the steady state inactivation curve (Lüttgau and Spiecker, 1979; Cota and Stefani, 1981; Bolaños et al., 1986). In the case of tetracaine, it is known that this drug, at 1-2 mM, impairs contractile activation (Vergara and Caputo, 1983) and causes a very
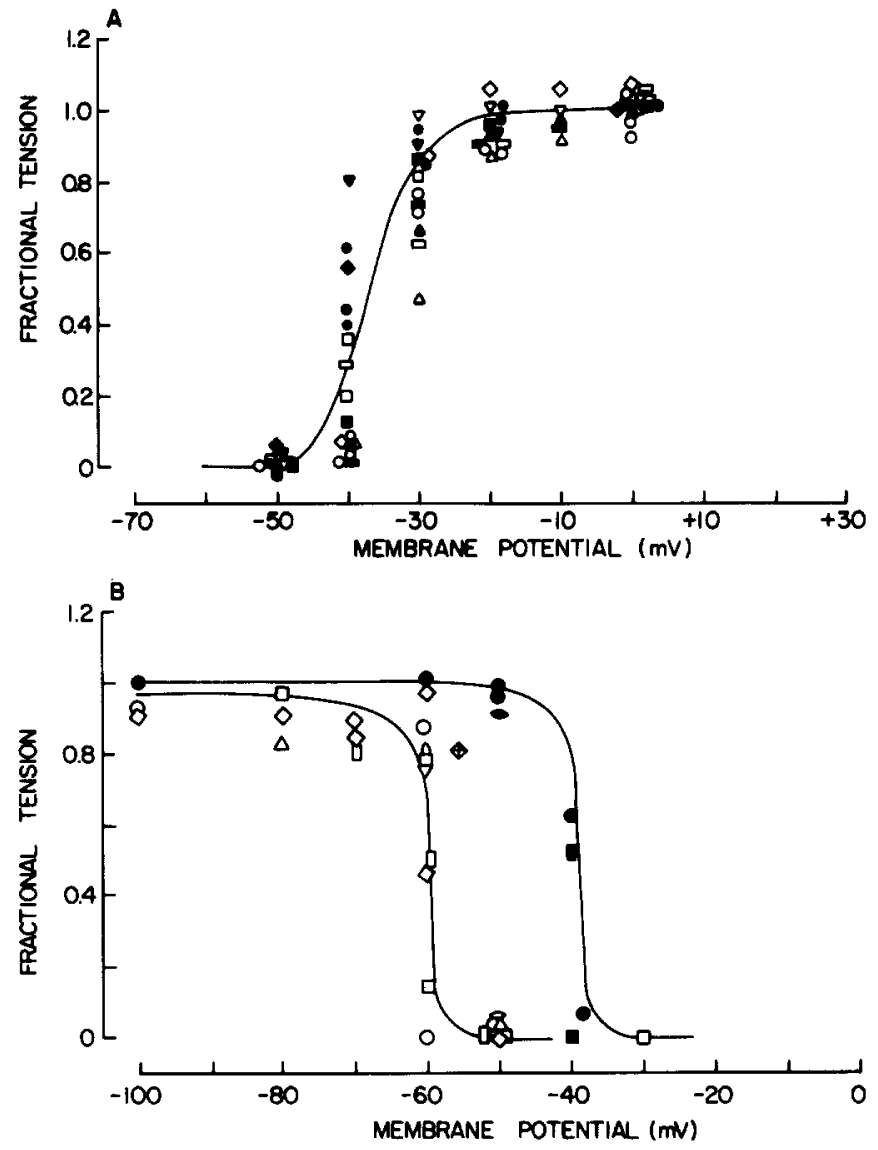

FIgURE 7. Effect of $100 \mu \mathrm{M}$ tetracaine on the activation $(A)$ and on the steady state inactivation curves $(B)$, obtained with several fibers. The graph in $A$ shows the relation between peak tension in response to $200-\mathrm{ms}$ depolarizing pulses and the membrane potential during the pulse. The graph in $B$ shows the extent of repriming plotted against the membrane potential during repriming. In the two graphs, each symbol corresponds to the same fiber (filled: NR; open: $100 \mu \mathrm{M}$ tetracaine).

large shift in the contractile threshold (Almers and Best, 1976). However, at lower concentrations $(0.1 \mathrm{mM})$, tetracaine does not seem to interfere with tension development, as shown in Fig. 5. A better demonstration of this is shown in Fig. $7 A$, which shows the tension-potential relation, obtained with several fibers in the absence and presence of the drug. In these experiments, test pulses of 200 ms duration and various amplitudes were used. In this and similar graphs, the 
tension values are normalized with respect to the maximum values obtained with each fiber in normal Ringer's solution. Each symbol corresponds to the same fiber. Fig. $7 B$ shows the effect of $0.1 \mathrm{mM}$ tetracaine on the steady state inactivation curve obtained with repriming experiments, in which contractures were elicited with pulses of $100 \mathrm{mV}$ amplitude and durations longer than $6 \mathrm{~s}$. The repriming period was $30 \mathrm{~s}$, which has been shown to be sufficient for complete repriming at $-100 \mathrm{mV}$ and at $20^{\circ} \mathrm{C}$ (Bolaños et al., 1986). The results shown here demonstrate that $0.1 \mathrm{mM}$ tetracaine does not affect contractile activation and causes an important shift in the steady state inactivation curve, as is also the case for low-calcium solutions.

\section{Effect of Dantrolene}

Dantrolene is known to interfere with calcium release from the SR (Putney and Bianchi, 1974); therefore, it was of interest to test its effects in fibers submitted to conditioning depolarizing ramps. Fig. 8 shows one such experiment. The

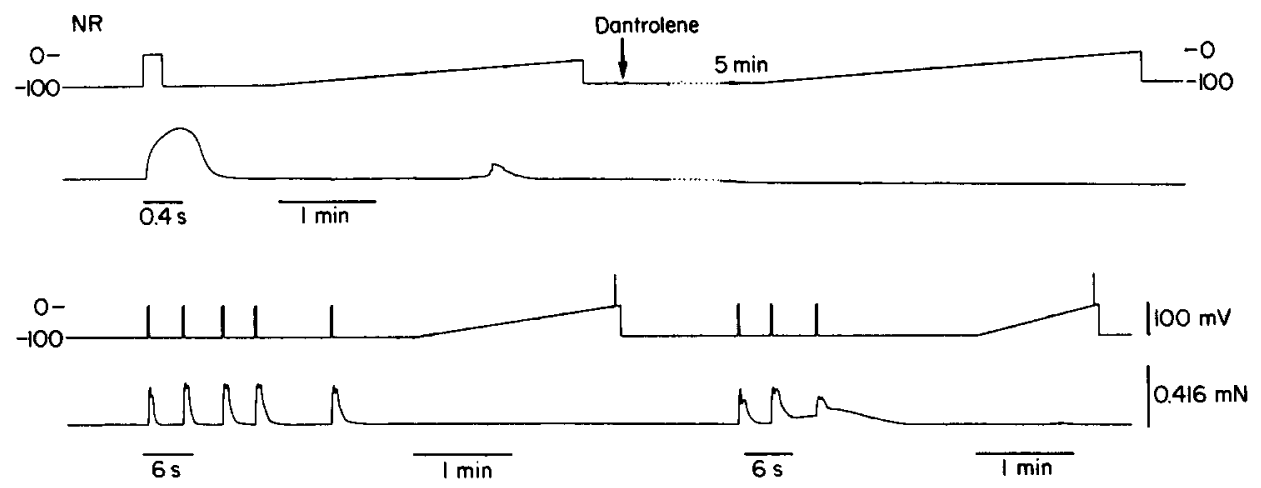

FIGURE 8. Effect of dantrolene $(50 \mu \mathrm{M})$ on a fiber sensitivity to depolarizing ramps. In the absence of the drug, a ramp of $0.4 \mathrm{mV} / \mathrm{s}$ did not cause complete inactivation, since a small response developed. In the presence of dantrolene, ramps of $0.4,0.75$, and $1.25 \mathrm{mV} / \mathrm{s}$ caused complete inactivation. Notice that after repolarization to $-100 \mathrm{mV}$ following depolarization with the ramp, the fiber recovered its contractile capacity despite the presence of dantrolene.

upper record shows that before dantrolene exposure, the fiber produced a small response during a $0.4-\mathrm{mV} / \mathrm{s}$ ramp; the other records, obtained in the presence of $50 \mu \mathrm{M}$ dantrolene, show that no responses were produced during $0.4-, 0.7-$, or $1.1-\mathrm{mV} / \mathrm{s}$ ramps. The figure also demonstrates that after repolarization to $-100 \mathrm{mV}$, the fiber recovered its contractile capacity, despite the presence of dantrolene. The results of several experiments with this compound are summarized in Fig. $6 B$, which demonstrates that in the presence of dantrolene, ramps of slopes up to $0.8 \mathrm{mV} / \mathrm{s}$ are effective in producing complete inactivation. Fig. $9 \mathrm{~A}$ demonstrates that the effects of dantrolene on the tension-potential relation depend on the stimulation pattern. In the graph, the fiber fractional tension, in response to 20 - or 200-ms pulses, in the presence or absence of $50 \mu \mathrm{M}$ dantrolene, is plotted against the fiber membrane potential during the pulses. It is evident 
that for the case of the longer-duration pulses, only a shift of the tension-voltage relation toward more positive potentials was caused by the drug, whereas for the shorter pulses, peak tension was reduced in the range of the membrane potential values tested. Fig. $9 B$ demonstrates that the drug at the same concentration did not modify the steady state inactivation curve obtained with repriming experiments.
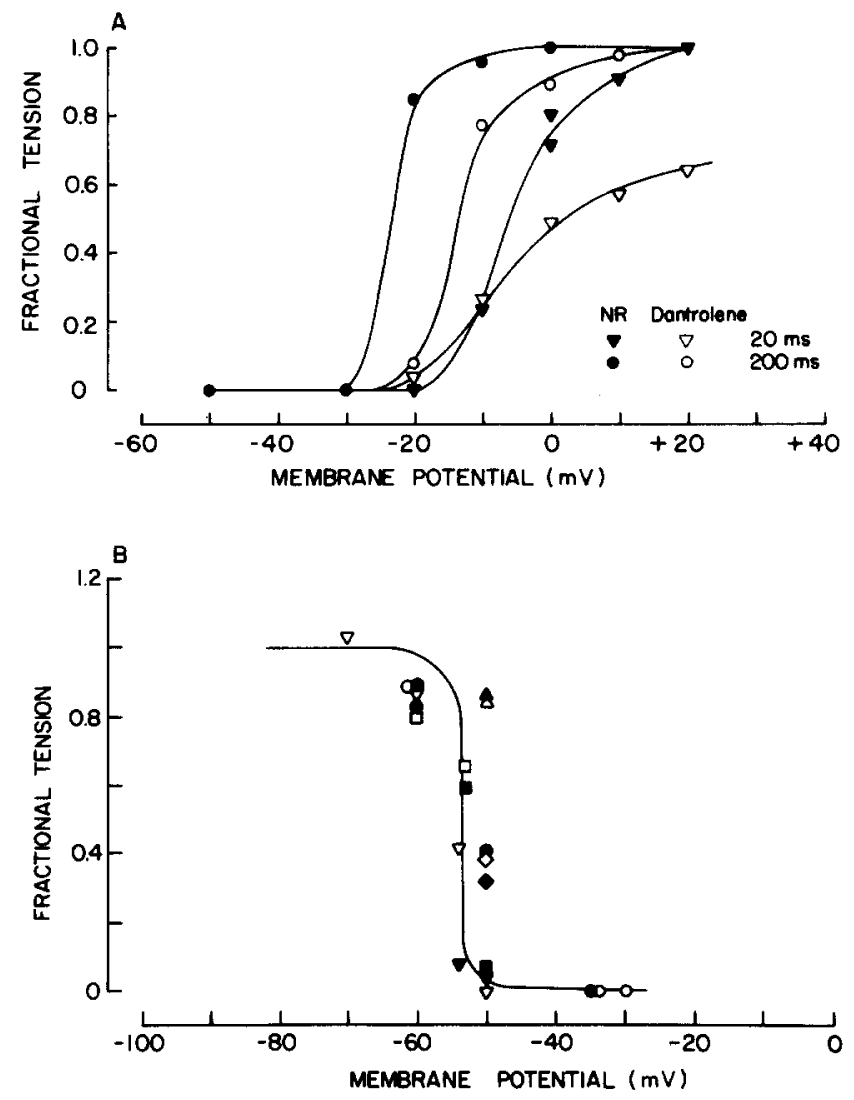

FIGURE 9. Effect of dantrolene on the activation $(A)$ and steady state inactivation curves $(B)$. In $A$, activation curves were obtained with two different pulse durations, 20 and $200 \mathrm{~ms}$. The drug induced a larger effect when the shorter pulse duration was used.

\section{Effects of D-600}

Lüttgau and Spiecker (1979) have shown that the calcium channel blocker D600 shortens the time course of potassium contractures in a way very similar to low-calcium solutions.

Fig. 10 shows an experiment in which the effect of D-600 $(50 \mu \mathrm{M})$ was tested. The upper record, obtained in normal Ringer's, shows first a response to a test pulse of $200 \mathrm{~ms}$ and $100 \mathrm{mV}$, and, immediately after, the response during a 
ramp of $0.4 \mathrm{mV} / \mathrm{slope}$, which was not effective in producing complete inactivation. The middle record, obtained after exposing the fiber to D-600, shows that the fiber was still able to respond to a test pulse, whereas no response was produced during a ramp of the same slope as before. The last record shows that faster slope ramps were also effective in producing inactivation.

An important aspect of this experiment is the failure of the fiber to generate tension in response to test pulses after the fiber has been repolarized to -100

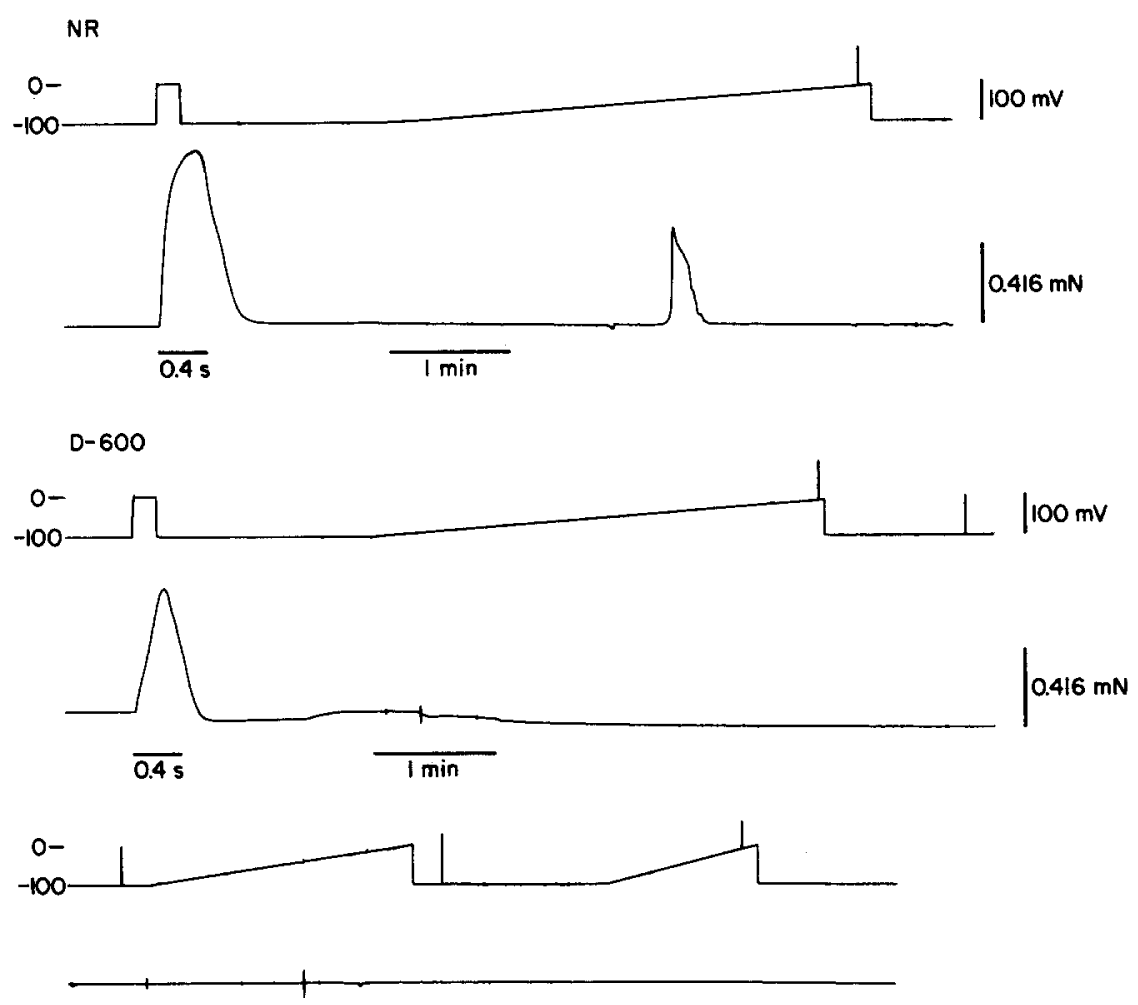

Figure 10. Effect of D-600 $(50 \mu \mathrm{M})$ on depolarizing ramp effectiveness. The upper record shows that with a ramp with slope $0.4 \mathrm{mV} / \mathrm{s}$, only partial inactivation was induced in this particular fiber. In the presence of D-600, only one contractile response could be obtained at the beginning, and after a $0.4-\mathrm{mV} / \mathrm{s} \mathrm{ramp}$, which was effective in inducing complete inactivation, no further responses could be obtained, either with faster ramps, or with test pulses applied either over the ramp or after repolarizing the fiber to $-100 \mathrm{mV}$.

$\mathrm{mV}$ after the first ramp in the presence of D-600. This indicates that after inactivation is achieved in the presence of the drug, it cannot be reversed by membrane repolarization, owing to a failure of the repriming process. This effect is undoubtedly a manifestation of the onset of the paralysis phenomenon originally described by Eisenberg et al. (1983) for fibers kept at low temperature, even though the present experiments were carried at $20^{\circ} \mathrm{C}$. Several other similar 
results are summarized in Fig. $6 \mathrm{~B}$. The experiments shown in this graph were performed without testing the fibers with pulses before the conditioning ramps. In other experiments, it was found that the D-600 effect could be reversed very slowly (tens of minutes); complete recovery could not be achieved, because of fiber damage.

Fig. 11 shows that D-600 does not affect the tension-voltage relation. It was not possible to obtain the steady state inactivation curve in the presence of D600 , since repriming was blocked. In order to obtain additional information on the inactivating properties of D-600, experiments of the type shown in Fig. 12 were carried out. Panel $A$ shows the effect of pulsing a fiber at two different duty cycles, in the absence (top records) and presence (bottom records) of D600 . In the first record, the fiber was stimulated with 500-ms pulses (duty cycle, 0.3 ), and no tension decay is observed. When stimulated with 1 -s pulses and a

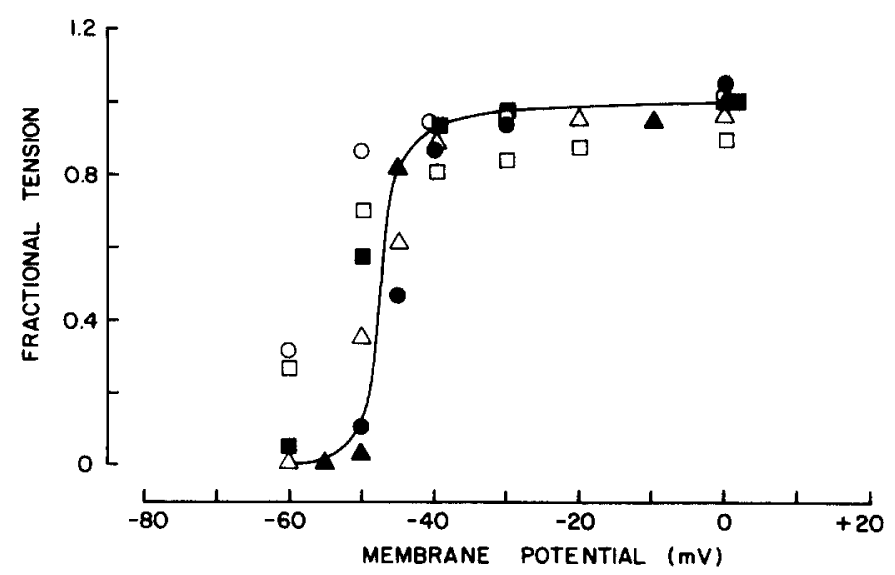

Figure 11. Effect of D-600 $(50 \mu \mathrm{M})$ on the tension-voltage relationship obtained with several fibers. In the graph, each symbol corresponds to the same fiber. Only a small reduction in the peak tension value was observed to occur in the presence of the drug.

0.5 duty cycle, tension decay was evident. After exposure to $50 \mu \mathrm{M}$ D-600, tension decay was appreciable with the first stimulation pattern and almost total with the second one. Panel $B$ shows that when another fiber was tested with a different pulse duration ( $100 \mathrm{~ms}$ or $1 \mathrm{~s})$, at the same duty cycle $(0.5)$, tension

FiguRE 12. (opposite) Effect of D-600 $(50 \mu \mathrm{M})$ on the contractile responses to repetitive stimulation with different duty cycle periods. Each section shows the results obtained with a different fiber. Panel $A$ shows that in the absence of the drug, contractile responses decayed very little or not at all when 0.5 -s pulses were applied at 1 -s intervals (duty cycle, 0.3 ) and somewhat more when 1 -s pulses were applied at the same interval (duty cycle, 0.5). In the presence of D-600, an appreciable fall of peak tension was observed with the first pulse pattern, and almost complete abolition of contraction was obtained with the second pulse pattern. Panel $B$ shows that after 26 min exposure to $50 \mu \mathrm{M}$ D-600 (at rest), a fiber can generate 


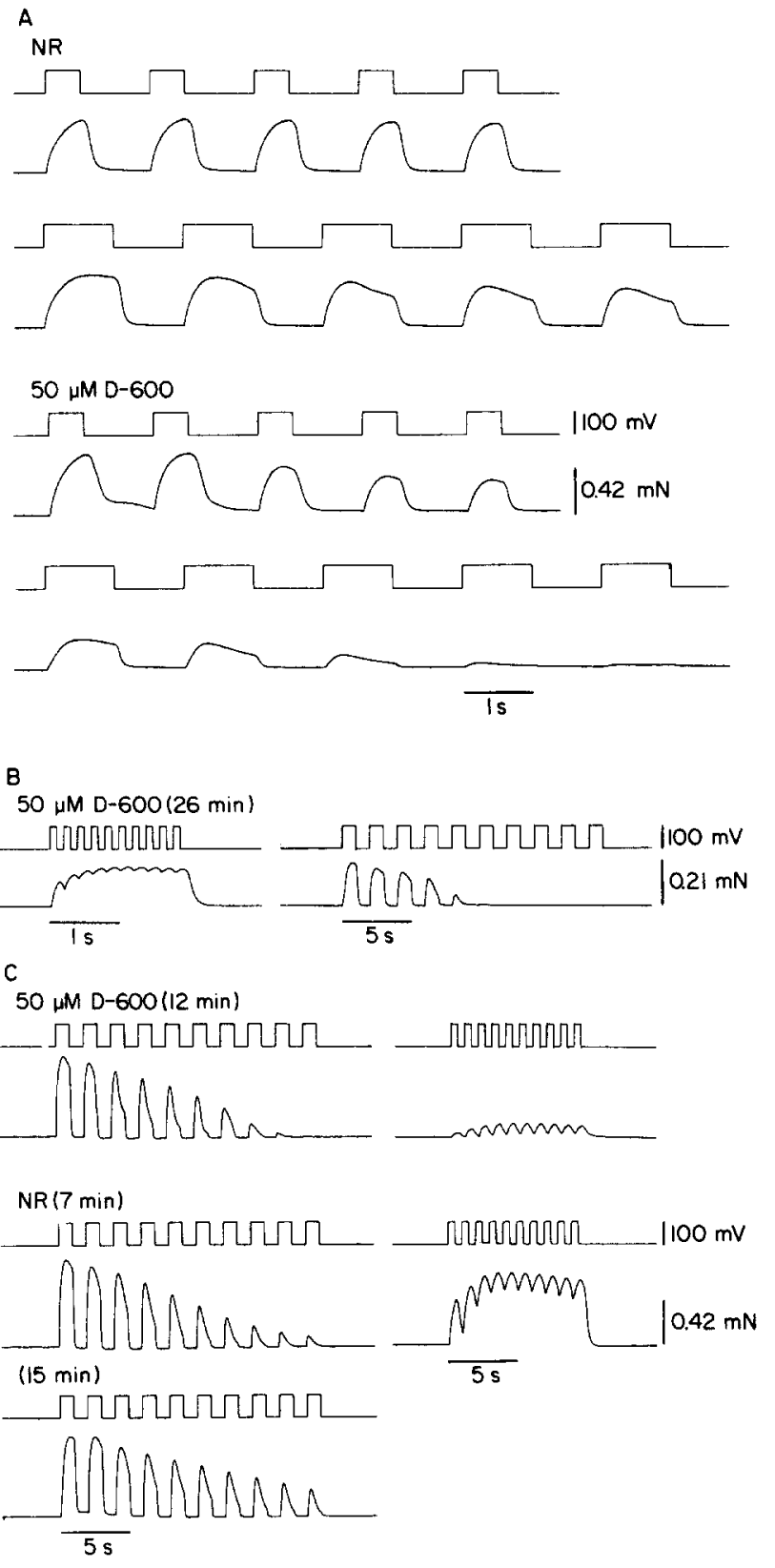

an almost fused tetanus, with no tension decay, when stimulated with 100 -ms stimuli at 100 -ms intervals (duty cycle, 0.5 ); however, a large tension decay was observed when the fiber was stimulated with 200 -ms pulses at 200 -ms intervals (duty cycle, 0.5 ). Finally, panel $C$ shows that once tension decayed to a low value as a result of a given stimulation pattern, it was not recovered while the drug was present. After removal of the drug, tension was recovered in a rather labile way since it can decay rapidly, depending on the stimulation pattern. 
decay occurred only with the longer pulse duration. Finally, panel $C$ shows that the tension decay observed in the presence of D-600 was reversed slowly after the drug was washed away. Interestingly, the first responses of the series obtained after returning to normal Ringer's appeared to be fully recovered; with further stimulation tension, they decayed when a longer pulse duration was used, bit not when shorter pulses were used.
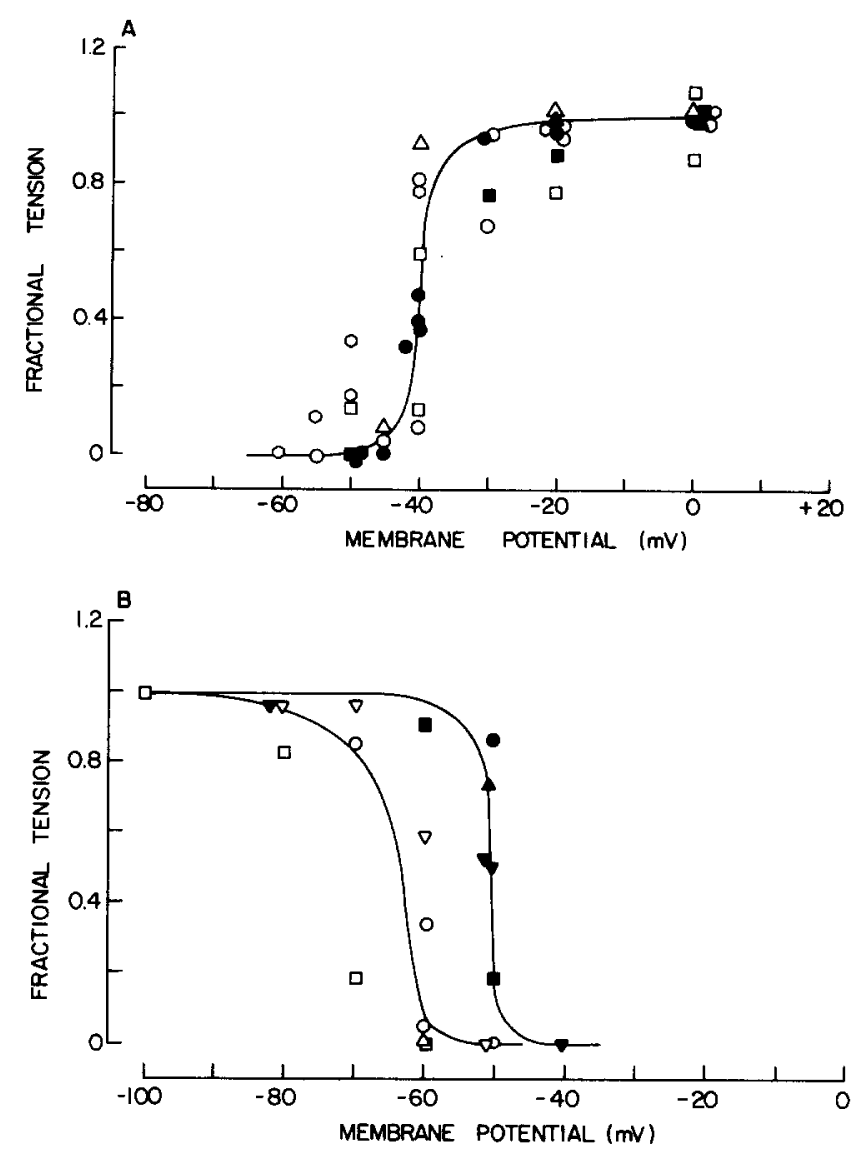

FigURE 13. Effect of nifedipine $(100 \mu \mathrm{M})$ on activation (upper graph) and steady state inactivation (lower graph) curves obtained with different fibers. Each symbol corresponds to the same fiber.

\section{Effect of Nifedipine}

In view of the effects of D-600, it was thought to be important to test the effects of another calcium blocker, so nifedipine was used (Cota and Stefani, 1981). Fig. $6 A$ summarizes the results obtained with ramp experiments, which demonstrate that nifedipine $(50 \mu \mathrm{M})$ was much less effective than D-600 in facilitating inactivation. Finally, Fig. 13 shows the effect of nifedipine $(100 \mu \mathrm{M})$ on the tension-voltage relationship $(A)$ and on the steady state inactivation curve $(B)$. The figure demonstrates a small shift in the latter and no effect on the former. 


\section{DISCUSSION}

The experiments described in this work demonstrate that it is possible to induce contractile inactivation without tension development in frog muscle fibers, and that various experimental treatments may differentially affect contractile parameters associated with contractile activation or inactivation. In a previous study (Caputo et al., 1984b), it was shown that when muscle fibers are conditioned with subthreshold depolarizing pulses, the onset of the inactivation process is characterized by a shortening of the time course of the contracture responses, followed by decreased tension output. The relation between contractile inactivation and membrane potential obtained in experiments in which inactivation was induced with subthreshold conditioning pulses and without previous contractile activation can be described by a curve very similar to the steady state inactivation curve that was obtained with repriming experiments (Hodgkin and Horowicz, 1960; Bolaños et al., 1986); this indicates that repriming can be considered the reverse operation of the mechanism responsible for inactivation. This apparently obviates the need to explain repriming on the basis of other processes, such as specific calcium translocations, although these may indeed occur (Curtis and Eisenberg, 1985). The effectiveness of slow conditioning depolarizing ramps and of subthreshold conditioning pulses in reducing or abolishing the ability of the fiber to develop tension cannot be taken as evidence that only the inactivation process is involved in this effect. It could also be due to a failure in the activation process, as is indicated by the experiments with dantrolene, which only affects activation. The experiments with low-calcium solutions, tetracaine at low concentration, D-600, and, to a lesser extent, nifedipine demonstrate that it is possible to affect the steady state inactivation curve without affecting the activation curve. This provides some indication that contractile activation and inactivation might be two separate processes. Before discussing the possible nature of the inactivation process, it is convenient to review the action of the pharmacological agents used in this work.

D-600 and dantrolene have been found to be most effective in increasing the fiber sensitivity for inactivation with slow depolarizing ramps. However, while dantrolene does not seem to affect the steady state inactivation curve, D-600 is effective in maintaining the fiber in a completely inactivated state, from which it recovers very slowly after removal of the drug. This state is similar to the paralysis state originally described by Eisenberg et al. (1983) under somewhat different conditions. Furthermore, while D-600 does not affect the activation curve, dantrolene affects it in a way that depends on the stimulation pattern, being most effective when short pulses are used; this agrees with previous work in which the effect of dantrolene on single twitches and tetani was studied (Putney and Bianchi, 1974). The effect of D-600 on the calcium release process does not appear to be related to its action as a calcium channel blocker (Curtis and Eisenberg, 1985; McCleskey, 1985). Even when used at a much higher concentration than that normally used for calcium antagonists $(50,100$, and 200 $\mu \mathrm{M}$ vs. $0.3 \mu \mathrm{M}$; Almers et al., 1981), nifedipine appears to be less effective than D-600 in experiments in which depolarizing ramp inactivation was studied, as well as in those in which repriming was studied. Finally, it is worth mentioning 
that D-600 appears to affect the inactivation mechanism in a way that depends on the depolarizing pulse duration, which suggests that it affects the inactivation mechanism while this is developing. This agrees with the effects of D-600 on the time course of potassium contractures (Lüttgau and Spiecker, 1979; Cota and Stefani, 1981). Other treatments that shorten the time course of potassium contractures, such as tetracaine at very low concentrations (100 or $200 \mu \mathrm{M}$ ) and low calcium (Caputo, 1972), also appear to be effective in increasing the fiber sensibility to inactivation, without affecting the activation curve. This is in agreement with the idea that the time course of the contracture responses is determined by the onset and time course of the inactivation process (Caputo, 1972).

In the preceding discussion, the inactivation process has been considered from a purely operational point of view, namely as the process that causes the spontaneous relaxation of contracture responses during sustained depolarization, and brings muscle fibers into a state of contractile refractoriness, which can later be reversed by membrane repolarization, through the repriming process. The experiments described here indicate that the inactivation process can be differentiated from the activation process by several experimental procedures. However, before discussing the mechanisms underlying the inactivation process, it is necessary to briefly review the current ideas about contractile activation. It is generally accepted that contractile activation depends on the opening of calcium channels in the SR membrane, triggered in response to a change in the fiber membrane potential and mediated by a voltage-sensitive process occurring at the level of the transverse tubule-sarcoplasmic reticulum (T-SR) junction (Grafe and Schatzman, 1984). The latter process, not yet elucidated, possibly involves intramembrane charge movement (Schneider and Chandler, 1974; Chandler et al., 1976). Although there is increasing evidence that, above contractile threshold, charge movement is closely related to the rate of calcium release (Rakowski et al., 1985; Melzer et al., 1986), the precise mechanism by which charge movement could control the gating of calcium channels is still not clear. The original hypothesis (Chandler et al., 1976) of fixed structures transmitting the signals (spanning the gap) between the transverse tubules and SR membranes could, in principle, be modified by considering the possibility of a chemical messenger diffusing between the two membrane systems. Calcium ions diffusing from the tubular lumen or from a restricted region adjacent to the T-SR junction, or, as has been recently proposed (Vergara et al., 1985), inositol-triphosphate, could serve as such a messenger; the availability of this messenger could be controlled either directly by the tubular membrane potential or by an intermediate, voltage-sensitive step (charge movement). Since the work of Heistracher and Hunt (1969), it has been postulated that calcium release could be controlled by a voltage-dependent conductance in the SR membrane, which, after being activated, would turn off with time, thus ending calcium release.

Recent evidence, however, indicates that the membrane potential across the SR membrane is probably vanishingly small or zero (Somlyo et al., 1977), and that the $S R$ membrane potential signals measured with dyes are more likely to be an effect, rather than the cause, of calcium release (Oetliker, 1982; Baylor et 
al., 1984). Thus, regulation of calcium conductance can hardly be regulated by the SR membrane potential in a way analogous to that of sodium and potassium conductances in excitable cell membranes. As pointed out by Baylor et al. (1984), calcium binding to the SR membrane could produce a surface potential that could affect the calcium permeability properties of the SR membrane. The binding of other compounds, such as inositol triphosphate (Vergara et al., 1985), could produce similar effects. On the basis of these speculations, inactivation could be explained in terms of calcium channels shutting off. Before considering some possibilities associated with this modality, one could think of other mechanisms to explain inactivation. The most obvious of these would be based on depletion of intracellular stores of calcium. According to this hypothesis, it would not be necessary to postulate two separate mechanisms for activation and inactivation, since activation would gradually terminate insofar as SR calcium stores would have been depleted. Since it is possible that during conditioning depolarizing ramps, a prolonged subthreshold release of calcium could deplete the intracellular stores, complete inactivation without activation could be compatible with such an idea. Similarly, repriming would be a consequence of calcium stores refilling. However, depletion of calcium during inactivation and its reaccumulation in the SR during repriming do not seem consistent with the marked voltage sensitivity of these processes and with their sensitivity to caffeine (Lüttgau and Oetliker, 1968; Caputo et al., 1981) and other drugs (this work). This cannot easily be explained by effects on the translocation of intracellular or extracellular calcium. In addition, repriming occurs in the virtual absence of external calcium (Lüttgau and Spiecker, 1981; Caputo, 1981; Cota and Stefani, 1983; Brum et al., 1985). Furthermore, it is known that the time course of the contracture response, which may be determined by the time course of the inactivation process, can be modulated by different pharmacological interventions in a way that is not consistent with the idea of a fixed amount of calcium available for release (Caputo, 1976, 1981; Bolaños et al., 1986). In spite of these arguments, one should still consider the possibility that a decrease in the SR calcium concentration occurs during prolonged contractile responses, thus reducing the driving force for calcium release. Another hypothesis could be based on decreased calcium influx caused by calcium depletion in the transverse tubules (Almers et al., 1981) or to inactivation of inward calcium currents during prolonged depolarizations (Cota et al., 1984). However, the possibility of obtaining normal responses and achieving repriming, in the virtual absence of external calcium and in the presence of agents that are known to block calcium channels, argues against the idea that contractile inactivation is caused by inactivation of inward calcium currents (Lüttgau and Spiecker, 1979; Caputo, 1981; Cota and Stefani, 1981; Brum et al., 1985; Bolaños et al., 1986). This conclusion agrees with the work of McCleskey (1985) demonstrating that calcium currents are not directly involved in the mechanism controlling calcium release.

Finally, one should consider the possibility that the phenomenon of contractile inactivation, as described in this work, is based on inactivation of calcium channels triggered by an independent process. For instance, it has been proposed (Baylor et al., 1983) that calcium increase in the myoplasm during contractile activation 
might be the signal for changing the SR calcium conductance during short pulses. As pointed out in the Introduction, this mechanism is possibly different from the slower inactivation considered here; indeed, from the present experiments, one would expect that, at most, only a subthreshold release of calcium would occur during contractile inactivation induced by depolarizing ramps. However, even in this case, a slow build-up of calcium at level of the SR membrane could occur, in which intracellular calcium buffers could participate. Another possibility is that inactivation of channels is mediated by changes occurring at the site of the T-SR junction, where the mechanism providing the voltage sensitivity to calcium release is possibly localized. For instance, immobilization of charge movement could lead to inactivation of calcium channels. The immobilization (inactivation) and repriming characteristics of charge movement are reminiscent of contractile inactivation and repriming, and indeed their similarity has been a factor for postulating a role for charge movement in depolarization-contraction coupling (Chandler et al., 1976). It is remarkable that some of the compounds that have been shown here to interfere with the process of contractile inactivation also affect intramembrane charge movement (Huang, 1982; Hui, 1983; Hui et al., 1984; Vergara and Caputo, 1983). The mechanism by which charge movement controls the closing of calcium channels during a prolonged depolarization is not clear, and in fact the same difficulty exists regarding the opening of the channels. The possibility that charge movement is associated with the liberation of a messenger (Vergara et al., 1985) should also be considered for the case of inactivation. Depletion of such a messenger could be the cause of the shutting off of calcium channels.

The authors are grateful to Ms. Rebeca Godoy for secretarial help, to Ms. Duwhya Otero for the illustrations, and to Messrs. Mora, Pérez, and Cazorla for building the experimental chambers and electronic equipment.

This work was supported by the Muscular Dystrophy Association and CONICIT fund S11148.

Original version received 14 February 1986 and accepted version received 31 July 1986.

\section{R E F E R E N C ES}

Almers, W., and P. M. Best. 1976. Effects of tetracaine on displacement currents and contraction of frog skeletal muscle. Journal of Physiology. 262:583-611.

Almers, W., R. Fink, and P. T. Palade. 1981. Calcium depletion in frog muscle tubules: the decline of calcium current under maintained depolarization. Journal of Physiology. 312:177207.

Baylor, S. M., W. K. Chandler, and M. W. Marshall. 1983. Sarcoplasmic reticulum calcium release in frog skeletal muscle estimated from arsenazo III calcium transients. Journal of Physiology. 344:625-666.

Baylor, S. M., W. K. Chandler, and M. W. Marshall. 1984. Calcium release and sarcoplasmic reticulum membrane potential in frog skeletal muscle fibres. Journal of Physiology. 348:209238. 
Bolaños, P., C. Caputo, and E. Velaz. 1986. Effects of calcium, barium and lanthanum on depolarization-contraction coupling in skeletal muscle fibres of Rana pipiens. Journal of Physiology. 370:39-60.

Brum, G., E. Rios, and J. Goldman. 1985. Calcium release and charge movement in skeletal muscle fibers exposed to $0 \mathrm{Ca}$ and long depolarizations. Biophysical Journal. 47:134a. (Abstr.)

Caputo, C. 1972. The time course of potassium contractures of single muscle fibres. Journal of Physiology. 233:483-505.

Caputo, C. 1976. The effect of caffeine and tetracaine on the time course of potassium contractures of single muscle fibres. Journal of Physiology. 255:191-207.

Caputo, C. 1981. Nickel substitution for calcium and the time course of potassium contractures of single muscle fibres. Journal of Muscle Research and Cell Motility, 2:167-182.

Caputo, C. 1983. Pharmacological investigations of excitation-contraction coupling. In Handbook of Physiology; Skeletal Muscle. L. D. Peachey and R. H. Adrian, editors. American Physiological Society, Bethesda, MD. 381-415.

Caputo, C., F. Bezanilla, and P. Horowicz. 1984a. Depolarization-contraction coupling in short frog muscle fibers. Journal of General Physiology. 84:133-154.

Caputo, C., P. Bolaños, and G. F. González. 1984b. Effect of membrane polarization on contractile threshold and time course of prolonged contractile responses in skeletal muscle fibers. Journal of General Physiology. 84:927-943.

Caputo, C., and P. Fernández de Bolaños. 1979. Membrane potential, contractile activation and relaxation rates in voltage clamped short muscle fibres of the frog. Journal of Physiology. 289:175-189.

Caputo, C., G. Gottschalk, and H. Ch. Lüttgau. 1981. The control of contraction activation by the membrane potential. Experientia. 37:580-581.

Chandler, W. K., R. F. Rakowski, and M. F. Schneider, 1976. Effects of glycerol treatment and maintained depolarization on charge movement in skeletal muscle. Journal of Physiology. 254:285-316.

Cota, G., L. N. Siri, and E. Stefani. 1984. Calcium channel inactivation in frog (Rana pipiens and Rana moctezuma) skeletal muscle fibres. Journal of Physiology. 354:99-108.

Cota, G., and E. Stefani. 1981. Effects of external calcium reduction on the kinetics of potassium contractures in frog twitch muscle fibres. Journal of Physiology. 317:303-316.

Curtis, B. A., and R. S. Eisenberg. 1985. Calcium influx in contracting and paralyzed frog twitch muscle fibers. Journal of General Physiology. 85:383-408.

Eisenberg, R. S., R. T. McCarthy, and R. L. Milton. 1983. Paralysis of frog skeletal muscle fibres by the calcium antagonist D-600. Journal of Physiology. 341:495-505.

Frankenhaeuser B., and J. Lannergren. 1967. The effect of calcium on the mechanical response of single twitch muscle fibres of Xenopus laevis. Acta Physiologica Scandinavica. 69:242-254.

Graf, F., and H.J. Schatzmann. 1984. Some effects of external calcium removal on pig striated muscle. Journal of Physiology. 349:1-13.

Heistracher, P., and C. C. Hunt. 1969. The relation of membrane changes to contraction in twitch muscle fibres. Journal of Physiology. 201:589-611.

Hodgkin, A. L., and P. Horowicz. 1960. Potassium contractures of single muscle fibres. Journal of Physiology. 153:386-403.

Huang, C. L.-H. 1982. Pharmacological separation of charge movement component in frog skeletal muscle. Journal of Physiology. 324:375-387.

Hui, C. S. 1983. Pharmacological studies of charge movement in frog skeletal muscle. Journal of Physiology. 337:509-529. 
Hui, C. S., R. L. Milton, and R. S. Eisenberg. 1984. Charge movement in skeletal muscle fibers paralyzed by the calcium-entry blocker D600. Proceedings of the National Academy of Sciences. $81: 2582-2585$.

Lüttgau, H. C. 1963. The action of calcium ions on potassium contractures of single muscle fibres. Journal of Physiology. 168:679-697.

Lütgau, H. C., and H. Oetliker. 1968. The action of caffeine on the activation of the contractile mechanism in striated muscle fibres. Journal of Physiology. 194:51-74.

Lüttgau, H. C., and W. Spiecker. 1979. The effects of calcium deprivation upon mechanical and electrophysiological parameters in skeletal muscle fibres of the frog. Journal of Physiology. 296:411-429.

McCleskey, E. W. 1985. Calcium channels and intracellular calcium release are pharmacologically different in frog skeletal muscle. Journal of Physiology. 361:231-249.

Melzer, W., E. Rios, and M. F. Schneider, 1984. Time course of calcium release and removal in skeletal muscle fibers. Biophysical Journal. 45:637-641.

Melzer, W., M. F. Schneider, B. J. Simon, and G. Szucz. 1986. Intramembrane charge movement and calcium release in frog skeletal muscle. Journal of Physiology. 373:481-51 1.

Oetliker, H. 1982. An appraisal of the evidence for a sarcoplasmic reticulum membrane potential and its relation to calcium release in skeletal muscle. Journal of Muscle Research and Cell Motility. 3:247-272.

Putney, J. W., and C. P. Bianchi. 1974. Site of action of dantrolene in frog sartorius muscle. Journal of Pharmacology and Experimental Therapeutics. 189:202-212.

Rakowski, R. F., P. M. Best, and M. R. James-Kracke. 1985. Voltage dependence of membrane charge movement and calcium release in frog skeletal muscle fibres. Journal of Muscle Research and Cell Motility. 6:403-433.

Schneider, M. F., and W. K. Chandler. 1974. Voltage dependent charge movement in skeletal muscle: a possible step in excitation-contraction coupling. Nature. 242:244-246.

Somlyo, A. V., H. Shuman, and A. P. Somlyo. 1977. Elemental distribution in striated muscle and the effects of hypertonicitiy. Electron probe analysis of cryo sections. Journal of Cell Biology. 74:825-857.

Vergara, J., and C. Caputo. 1983. Effects of tetracaine on change movements and channel signals in frog skeletal muscle fibers. Proceedings of the National Academy of Sciences. 80:14771481 .

Vergara, J., R. Y. Tsien, and M. Delay. 1985. Inositol 1,4,5-triphosphate: a possible chemical link in excitation-contraction coupling in muscle. Proceedings of the National Academy of Sciences. 82:6352-6356. 